\title{
Evaluación de la utilidad de la vitamina D como predictor de mortalidad en pacientes con COVID-19
}

\author{
Erick J. Martínez-Rodríguez, ${ }^{*}$ Juan Gutiérrez-Mejía, ${ }^{1}$ Camilo Ríos-Castañeda, ${ }^{2}$ Sonia Rojas-Maya ${ }^{3}$ y \\ Adrián Soto-Mota ${ }^{1}$ \\ ${ }^{1}$ Departamento de Reacción Hospitalaria para Desastres, Instituto Nacional de Ciencias Médicas y Nutrición "Salvador Zubirán"; ${ }^{2}$ Departamento \\ de Neuroquímica, Instituto Nacional de Neurología y Neurocirugía "Manuel Velasco Suárez"; ${ }^{3}$ Departamento de Laboratorio Clínico, Instituto \\ Nacional de Ciencias Médicas y Nutrición "Salvador Zubirán". Ciudad de México, México
}

\section{Resumen}

Introducción: Una de las funciones de la vitamina $D$ es regular la respuesta inflamatoria del epitelio respiratorio; por ello, la deficiencia de esa vitamina en el contexto de COVID-19 podría constituir un biomarcador preditivo del desenlace de COVID-19. Objetivo: Evaluar la utilidad de la vitamina D para predecir la mortalidad en pacientes con COVID-19. Métodos: Estudio observacional y retrospectivo en el que se incluyeron 154 pacientes con diagnóstico de COVID-19, de los cuales 111 sobrevivieron y 43 fallecieron. En todos se determinó la concentración de vitamina $D$. Resultados: Se obtuvo un valor log-rank de $p<0.032$ para la supervivencia al utilizar la concentración de vitamina $D$ como variable categórica ( $\leq 20 \mathrm{ng} / \mathrm{mL}$ y $>20 \mathrm{ng} / \mathrm{mL}$ ). Mediante análisis proporcional de Cox se encontró que la edad y concentración de vitamina $D$ mostraron ser factores de riesgo asociados a la mortalidad en pacientes con COVID-19 (edad: $H R=1.036, I C 95 \%=1.016-1.058, p<0.001$; vitamina D: $H R \leq 20 \mathrm{ng} / \mathrm{mL}$ y > $20 \mathrm{ng} / \mathrm{mL}=0.478$, IC $95 \%=0.237-0.966, p<0.040)$. Conclusión: La edad y la concentración de vitamina $D$ constituyeron factores predictivos de mortalidad en pacientes infectados por COVID-19.

PALABRAS CLAVE: Vitamina D. COVID-19. Respuesta inflamatoria del epitelio respiratorio. Factor predictivo.

\section{Evaluation of the usefulness of vitamin $D$ as a predictor of mortality in patients with COVID-19}

\section{Abstract}

Introduction: One of the functions of vitamin $D$ is to regulate respiratory epithelium inflammatory response; therefore, deficiency of this vitamin in the context of COVID-19 could constitute a predictive biomarker of the disease outcome. Objective: To evaluate the usefulness of vitamin $D$ for predicting mortality in patients with COVID-19. Methods: Observational, retrospective study in which 154 patients diagnosed with COVID-19 were included, out of whom 111 survived and 43 died. Vitamin D concentration was determined in all of them. Results: $A$ log-rank $p$-value $<0.032$ was obtained for survival when vitamin $D$ concentration was used as a categorical variable ( $\leq 20 \mathrm{ng} / \mathrm{mL}$ and $>20 \mathrm{ng} / \mathrm{mL}$ ). On Cox proportional analysis, age and vitamin $D$ concentration were shown to be risk factors associated with mortality in patients with COVID-19 (age: HR = 1.036, 95\% Cl = 1.016-1.058, $p<0.001$; vitamin D: $H R[\leq 20 \mathrm{ng} / \mathrm{mL}$ and $>20 \mathrm{ng} / \mathrm{mL}]=0.478,95 \% \mathrm{Cl}=0.237-0.966, p<0.040)$. Conclusion: Age and vitamin D concentration were predictive factors for mortality in COVID-19-infected patients.

KEYWORDS: Vitamin D. COVID-19. Respiratory epithelium inflammatory response. Predictive factor.

Correspondencia:

*Erick J. Martínez-Rodríguez

E-mail: erickmarrod99@gmail.com

0016-3813/@ 2021 Academia Nacional de Medicina de México, A.C. Publicado por Permanyer. Este es un artículo open access bajo la licencia CC BY-NC-ND (http://creativecommons.org/licenses/by-nc-nd/4.0/).
Fecha de recepción: 21-06-2021

Fecha de aceptación: 08-07-2021 


\section{Introducción}

Actualmente se conoce que COVID-19 es altamente contagiosa, además de ser potencialmente letal. Las personas mayores de 60 años, y particularmente quienes tienen comorbilidades como diabetes, hipertensión, enfermedades cardiovasculares, cáncer y obesidad tienen un mayor riesgo de severidad de esta enfermedad. ${ }^{1}$

Es interesante mencionar que las regiones más afectadas por COVID-19 comparten condiciones climáticas similares y que la prevalencia de la enfermedad pudiera estar relacionadas con bajos niveles de vitamina $\mathrm{D}$ en las poblaciones. La tasa de hospitalizaciones también ha mostrado una fuerte correlación con la latitud de los países. ${ }^{2}$

La relevancia de la deficiencia o suplementación de vitamina $D$ en esta enfermedad ha sido controversial. Algunos estudios aseguran que la suplementación de vitamina $\mathrm{D}$ podría ser un factor protector, ${ }^{3,4}$ pero otros no recomiendan su uso. ${ }^{5}$ Por un lado, se conoce que la vitamina $D$ estimula la respuesta de inmunidad innata y modula la inmunidad adquirida, además de que desempeña un papel importante en el desenlace de las infecciones respiratorias. ${ }^{6}$ También se conoce que la vitamina $D$ puede atenuar la respuesta hiperinflamatoria, reducir la producción de citocinas proinflamatorias y acelerar el proceso de reparación de los epitelios afectados, principalmente el tejido pulmonar. ${ }^{7}$

Por otro lado, la deficiencia de vitamina $D$ se ha asociado a incremento de los episodios trombóticos en pacientes con COVID-19. ${ }^{8}$ Además, un estudio reciente demostró que la mortalidad por COVID-19 es mayor en pacientes con concentraciones de vitamina $D$ inferiores a $12 \mathrm{ng} / \mathrm{mL} .{ }^{9}$ Sin embargo, en otra investigación, la suplementación controlada de esta vitamina en pacientes con COVID-19 no mostró diferencias en la mortalidad ni en los días de hospitalización. ${ }^{10}$

La deficiencia de vitamina $D$ también es común en personas de piel oscura y con pocas horas de exposición a radiación solar. Estos factores han sido estudiados y asociados a mayor riesgo de gravedad de COVID-19.2,11 Vale la pena resaltar que la deficiencia de vitamina $D$ es más común en las personas institucionalizadas, como los presos y los ancianos confinados en centros geriátricos. ${ }^{12}$

El sexo también ha mostrado desempeñar un papel importante en el desenlace de COVID-19; algunos estudios han propuesto que existe una mayor letalidad de la enfermedad en hombres versus mujeres. ${ }^{13}$ Además, la deficiencia de vitamina $D$ ha mostrado interacción significativa con la edad ${ }^{14,15}$ Las variables anteriores podrían predisponer a la muerte. ${ }^{15}$

Existen varios biomarcadores para predecir la gravedad de COVID-19 como la proteína $C$ reactiva, la creatinina, comorbilidades particulares, etcétera: ${ }^{16} \sin$ embargo, la vitamina $D$ no ha sido estudiada ni propuesta en alguna escala como posible biomarcador predictivo de mortalidad en pacientes con COVID-19. Podría constituir un predictor de otros factores de riesgo causales, además de que su medición sería útil y de fácil acceso. No obstante, los valores de corte son variables y tienen que ser conocidos y estudiados en población mexicana.

Mencionado lo anterior, la gravedad de la infección de COVID-19 y su relación con la deficiencia de vitamina $D$ podría estar predispuesta por factores alimentarios, ambientales, sociales y geográficos. Hasta el momento no se ha estudiado un punto de corte específico para evaluar la deficiencia de vitamina $D$ en población mexicana. En este estudio investigamos el potencial de la vitamina $D$ como factor pronóstico de mortalidad en pacientes mexicanos con COVID-19.

\section{Métodos}

Se trató de un estudio retrospectivo observacional realizado en el Instituto Nacional de Nutrición y Ciencias Médicas "Salvador Zubirán", Ciudad de México, el cual fue aprobado por el comité de ética del mismo hospital con número de registro DMC3369-20-20-1-1. El estudio incluyó a 154 pacientes consecutivos con COVID-19, 54 mujeres y 100 hombres, atendidos entre diciembre de 2020 y febrero de 2021, de los cuales 111 sobrevivieron y 43 fallecieron. Todos los pacientes fueron confirmados con RT-PCR. Los niveles de vitamina $D$ fueron cuantificados en el laboratorio clínico del mismo hospital al momento del ingreso hospitalario.

Los datos de los pacientes desde el inicio de la enfermedad hasta el egreso o la defunción fueron recopilados del expediente clínico.

El tiempo de supervivencia se consideró a partir del primer día de ingreso al servicio de urgencias hasta la defunción o el egreso del paciente.

El número de participantes se eligió por viabilidad de acuerdo con lo establecido por Bacchetti. ${ }^{17,18} \mathrm{Se}$ recolectó la información de 154 pacientes. Para el análisis estadístico se utilizó el programa SPSS versión 25. Para el análisis bivariado se utilizó la prueba 
de $t$ de Student y $\chi^{2}$; las variables del estudio fueron comparadas entre grupos independientes (supervivientes versus fallecidos). La curva de Kaplan-Meier fue construida para analizar la supervivencia. Se utilizó un modelo proporcional de riesgo de Cox multivariado para determinar los factores predictivos de la enfermedad. Para estos últimos se diseñó una variable categórica en la que el punto de corte fue establecido por la sociedad de endocrinología clínica: ${ }^{19}$ concentración de vitamina $D \leq 20 \mathrm{ng} / \mathrm{mL}$ $y>20 \mathrm{ng} / \mathrm{mL}$. La diferencia estadística fue aceptada en todos los análisis con un valor de $p<0.05$.

\section{Resultados}

Fueron incluidos 154 pacientes con COVID-19, 43 de ellos fallecieron y 111 sobrevivieron. En el análisis bivariado se encontró que la media de edad fue estadísticamente mayor en quienes fallecieron $(61.8 \pm 13.30$ años), en comparación con los supervivientes (51.44 $\pm 14.59, p<0.001)$. La concentración de vitamina $D$ no mostró ser estadísticamente significativa en los pacientes supervivientes $(18.60 \pm 7.65)$ versus los que fallecieron $(17.27 \pm 9.60, p=0.37)$. Tampoco se encontraron diferencias significativas en sexo, índice de masa corporal y días de hospitalización (Tabla 1).

Los pacientes fueron reclasificados en dos grupos conforme la cuantificación de vitamina $D(\leq 20 \mathrm{ng} /$ $\mathrm{mL}$ y $>20 \mathrm{ng} / \mathrm{mL}$ ) de acuerdo con lo establecido por una sociedad de endocrinología y estudios realizados en población mexicana. ${ }^{19,20}$ Las curvas de supervivencia de Kaplan-Meier mostraron que los pacientes con concentración de vitamina $\mathrm{D} \leq 20 \mathrm{ng} / \mathrm{mL}$ tuvieron una mayor probabilidad de defunción versus aquellos con concentración superior (log-rank, $p<0.032$ ), como se aprecia en la Figura 1.

El análisis multivariado de supervivencia con modelos de regresión de Cox mostró la influencia de la vitamina $D$ en el desenlace de los pacientes con COVID-19. La edad $(p<0.001)$ y la vitamina $D$ como variable categórica $(p<0.040)$ fueron factores asociados a mortalidad (edad: $\mathrm{HR}=1.036, \mathrm{IC}$ $95 \%=1.016-1.058, \mathrm{p}<0.000$; vitamina $\mathrm{D}, \mathrm{HR}=0.478$, IC $95 \%=0.237-0.966, p<0.04)$ (Tabla 2).

\section{Discusión}

Debido a que los puntos de corte para deficiencia e insuficiencia de vitamina $D$ en México han sido establecidos con base en estudios realizados en pacientes con alteraciones del metabolismo óseo y mineral, ${ }^{20}$ en este
Tabla 1. Variables estudiadas en pacientes con COVID-19, quienes fueron agrupados según el desenlace de la enfermedad

\begin{tabular}{|c|c|c|c|c|c|c|}
\hline \multirow[t]{2}{*}{ Variable } & \multirow{2}{*}{\multicolumn{2}{|c|}{$\frac{\text { Defunción }}{\text { Media } \pm \text { DE }}$}} & \multirow{2}{*}{\multicolumn{2}{|c|}{$\begin{array}{c}\text { Supervivientes } \\
\text { Media } \pm \text { DE }\end{array}$}} & \multirow[t]{2}{*}{$\mathbf{p}^{*}$} & \multirow[t]{2}{*}{$\chi^{2} / Z$} \\
\hline & & & & & & \\
\hline Edad & \multicolumn{2}{|c|}{$61.8 \pm 13.30$} & \multicolumn{2}{|c|}{$51.44 \pm 14.59$} & 0.000 & \\
\hline $\begin{array}{l}\text { Índice de masa } \\
\text { corporal }\end{array}$ & \multicolumn{2}{|c|}{$28.99 \pm 6.48$} & \multicolumn{2}{|c|}{$29.81 \pm 5.21$} & NS & \\
\hline $\begin{array}{l}\text { Días de } \\
\text { hospitalización }\end{array}$ & \multicolumn{2}{|c|}{$18.55 \pm 11.90$} & \multicolumn{2}{|c|}{$19.54 \pm 17.04$} & NS & \\
\hline \multirow{2}{*}{$\begin{array}{l}\text { Concentración de } \\
\text { vitamina D } \\
(\mathrm{ng} / \mathrm{mL})\end{array}$} & \multicolumn{2}{|c|}{$17.27 \pm 9.60$} & \multicolumn{2}{|c|}{$18.60 \pm 7.65$} & \multirow[t]{2}{*}{ NS } & \\
\hline & $\mathrm{n}$ & $\%$ & $\mathrm{n}$ & $\%$ & & \\
\hline $\begin{array}{l}\text { Sexo } \\
\text { Femenino } \\
\text { Masculino }\end{array}$ & $\begin{array}{l}16 \\
27\end{array}$ & $\begin{array}{l}10.38 \\
17.53\end{array}$ & $\begin{array}{l}38 \\
73\end{array}$ & $\begin{array}{l}24.67 \\
47.40\end{array}$ & & $\begin{array}{l}\text { NS } \\
\text { NS }\end{array}$ \\
\hline
\end{tabular}

Tabla 2. Análisis multivariado de supervivencia para determinar la influencia de la vitamina $D$ en el desenlace de los pacientes con COVID-19

\begin{tabular}{|c|c|c|c|c|c|c|}
\hline \multirow[t]{2}{*}{ Modelo } & \multirow[t]{2}{*}{ Variable } & \multirow[t]{2}{*}{ B } & \multirow[t]{2}{*}{ Sig. } & \multirow[t]{2}{*}{$\operatorname{Exp}(B)$} & \multicolumn{2}{|c|}{ IC $95 \% \operatorname{Exp}(B)$} \\
\hline & & & & & Inferior & Superior \\
\hline \multirow[t]{2}{*}{1} & Sexo & -0.093 & 0.765 & 0.907 & 0.47 & 1.724 \\
\hline & Edad & 0.033 & 0.002 & 1.033 & 1.012 & 1.055 \\
\hline \multirow[t]{3}{*}{2} & Sexo & -0.379 & 0.265 & 0.685 & 0.352 & 1.332 \\
\hline & Edad & 0.036 & 0.001 & 1.036 & 1.016 & 1.058 \\
\hline & $\begin{array}{l}\text { Vitamina } \\
\text { D }\end{array}$ & -0.738 & 0.040 & 0.478 & 0.237 & 0.966 \\
\hline
\end{tabular}

Modelos de Cox multivariado.

trabajo se decidió utilizar el punto de corte determinado por una sociedad de endocrinología. ${ }^{19}$ En México se considera que hay deficiencia de vitamina $D$ en sangre cuando esta se encuentra por debajo de $30 \mathrm{ng} / \mathrm{mL}$ y la insuficiencia por debajo de $20 \mathrm{ng} / \mathrm{mL} .{ }^{20}$

Nuestros resultados concuerdan con los de estudios previos en los cuales la edad y las concentraciones inferiores de vitamina $D$ se asociaron a mayor riesgo de mortalidad por COVID-19.9,21,22

En un estudio de pacientes hospitalizados por COVID-19, la insuficiencia de vitamina $D<30 \mathrm{ng} / \mathrm{mL}$ estuvo presente en $75 \%$ de la población y en $85 \%$ de todos los pacientes que requirieron cuidados intensivos. ${ }^{23}$ 


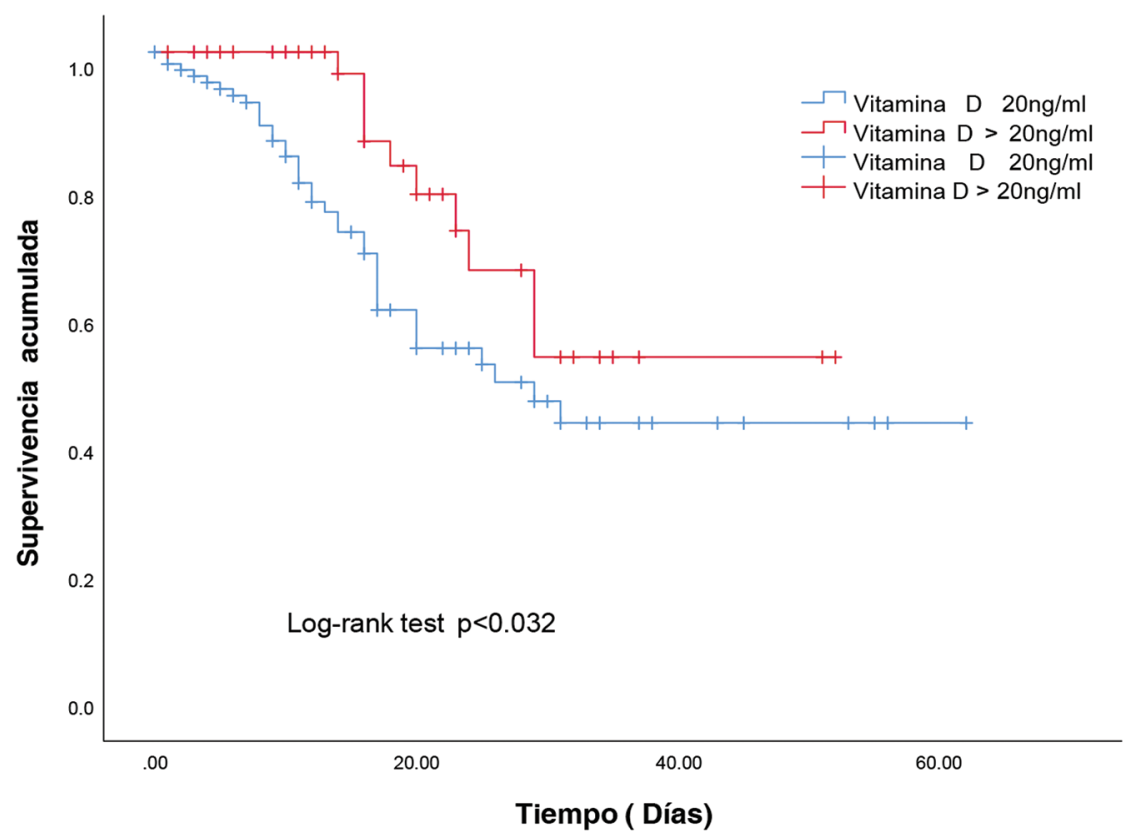

Figura 1. Análisis de supervivencia de Kaplan-Meier de acuerdo con niveles de vitamina D. La deficiencia de vitamina $D$ se asoció a reducción de la supervivencia (log rank, $p=0.032$ ).

D’Avolio et al. evaluaron la concentración de vitamina $D$ en 107 voluntarios; encontraron que la media de concentración de vitamina $D$ fue de $22.2 \mathrm{ng} / \mathrm{mL}$ en sujetos sin COVID-19, mientras que la media en 27 individuos con infección por SARS-CoV-2 confirmada por PCR fue $11.1 \mathrm{ng} / \mathrm{mL} .{ }^{24}$ Se debe mencionar que en ambos grupos (fallecidos versus supervivientes) de la investigación que presentamos, la media de concentración de vitamina $D$ se encontraba en valores de insuficiencia; sin embargo, las concentraciones $\leq 20 \mathrm{ng} / \mathrm{mL}$ se asociaron a mayor mortalidad en los pacientes con COVID-19, lo que también ha sido planteado en estudios similares, en los cuales se encontró que niveles $<30 \mathrm{ng} / \mathrm{mL}$ y la edad $\geq 65$ años se asociaron a mayor mortalidad por COVID-19..$^{25,26}$

Algunas investigaciones han mostrado que los niveles de vitamina $D$ no determinan el desenlace de COVID-19 y que la vitamina $D$ no desempeña un factor pronóstico de la mortalidad. ${ }^{27}$ Las variables incluidas en los diferentes modelos matemáticos, los puntos de corte utilizados o las medidas antes o después de una suplementación pueden marcar las diferencias en los resultados. ${ }^{10}$

En los modelos proporcionales de Cox ajustados por edad, sexo y vitamina $D$, nuestros resultados mostraron que la edad y la vitamina $D$ constituyeron factores estadísticamente significativos para mortalidad por COVID-19. En el modelo 2, la edad y la vitamina D mostraron una asociación estadísticamente significativa mayor que en el modelo número 1 , en el que no se consideró la vitamina D. En ninguno modelo se identificó asociación con el sexo (Tabla 2).

Peruzzu et al. tampoco encontraron diferencias significativas de vitamina $D$ entre los sexos, lo que sugiere que la presencia de factores biológicos u hormonales, particularmente los estrógenos y la testosterona, podrían subyacer en estas diferencias. ${ }^{28}$

Es posible que la concentración de vitamina $D$ en este trabajo constituyera un factor de riesgo para la severidad de la enfermedad. Sería tentador plantear la hipótesis de que la vitamina $D$ y los estrógenos afectan las diferencias de sexo en los resultados de los pacientes con COVID-19. ${ }^{29}$ Es importante agregar que la edad y las concentraciones de vitamina $D$ apuntan a un efecto potencial en el desenlace de los pacientes con COVID-19. Tanto los pacientes supervivientes como los fallecidos mostraron insuficiencia de vitamina $D$, lo que podría sugerir diversas preguntas de investigación asociadas a la alimentación, el metabolismo e, incluso, factores geográficos (latitud)..$^{30,31}$

Lanham et al. analizaron la severidad de COVID-19 asociada a niveles de vitamina $D$ en población europea. Concluyeron que en los países en los que se registra alta deficiencia de vitamina $D$, esta se asoció a altas tasas de infección y muerte. ${ }^{32}$ 
En un estudio reciente en México en el que se evaluaron variables como la temperatura, la humedad y la radiación ultravioleta y su asociación con la incidencia de trasmisión y muerte por COVID-19, se mostró que la radiación ultravioleta tiene un efecto fisiológico que repercute en la reducción de la mortalidad por COVID-19, lo que se explicó por la deficiencia de la vitamina $D .{ }^{33}$

También es posible que algunas vías metabólicas, factores inmunológicos o sociales sean relevantes y determinantes en el proceso de infección por SARSCoV-2 y la consecuente mortalidad. ${ }^{34}$ Sin embargo, si estudios clínicos bien controlados con suplementación de vitamina $D$ no mostraron diferencias estadísticas en el desenlace de COVID-19, entonces la concentración de vitamina $D$ podría ser, por lo menos, un buen predictor de severidad que ningún modelo predictivo validado para esa enfermedad incluye hasta el momento.

\section{Conclusión}

En este estudio, la edad y la concentración de vitamina $D$ mostraron ser posibles factores predictivos de mortalidad en pacientes por COVID-19, por lo que podrían ser consideradas como factores independientes para el riesgo de mortalidad de esta enfermedad. Sin embargo, se requieren estudios complementarios para evaluar la interacción de otras variables asociadas a la regulación de vitamina $\mathrm{D}$.

\section{Agradecimientos}

Agradecemos infinitamente al Instituto Nacional de Ciencias Médicas y Nutrición "Salvador Zubirán" por permitirnos revisar los expedientes electrónicos.

\section{Financiamiento}

La presente investigación no ha recibido ninguna beca específica de agencias de los sectores públicos, comercial o sin ánimo de lucro.

\section{Conflicto de intereses}

Los autores declaran no tener conflicto de intereses.

\section{Responsabilidades éticas}

Protección de personas y animales. Los autores declaran que para esta investigación no realizaron experimentos en seres humanos ni en animales.

Confidencialidad de los datos. Los autores declaran que siguieron los protocolos de su centro de trabajo sobre la publicación de datos de pacientes.

Derecho a la privacidad y consentimiento informado. Los autores declaran que en este artículo no aparecen datos de pacientes.

\section{Bibliografía}

1. Cascella M, Rajnik M, Cuomo A, Dulebohn SC, Di Napoli R. Features, evaluation and treatment coronavirus (COVID-19). EE. UU.: StatPearls Publishing; 2020.

2. Abraham J, Dowling K, Florentine S. Can optimum solar radiation exposure or supplemented vitamin D intake reduce the severity of COVID-19 symptoms? Int J Environ Res Public Health. 2021;18:1-21.

3. Grant WB, Lahore H, McDonnell SL, Baggerly CA, French CB, Aliano $\mathrm{JL}$, et al. Evidence that vitamin $\mathrm{D}$ supplementation could reduce risk of influenza and COVID-19 infections and deaths. Nutrients. 2020;12:988.

4. Ali N. Role of vitamin D in preventing of COVID-19 infection, progression and severity. J Infect Public Health. 2020;13:1373-1380.

5. Zemb P, Bergman P, Camargo CA, Cavalier E, Cormier C, Courbebaisse M, et al. Vitamin D deficiency and the COVID-19 pandemic. J Glob Antimicrob Resist. 2020;22:133-134.

6. Cashman KD, Dowling KG, Škrabáková Z, Gonzalez-Gross M, Valtueña J, Moreno L, et al. Vitamin D deficiency in Europe: pandemic? Am J Clin Nutr. 2016;103:1033-1044.

7. Mohan M, Cherian JJ, Sharma A. Exploring links between vitamin D deficiency and COVID-19. PLoS Pathog. 2020;16:e1008874.

8. Kenneth-Weir E, Thenappan T, Bhargava M, Chen Y. Does vitamin D deficiency increase the severity of COVID-19? Clin Med. 2020;20:e107-e108.

9. Radujkovic A, Hippchen T, Tiwari-Heckler S, Dreher S, Boxberger M, Merle U. Vitamin D deficiency and outcome of COVID-19 patients. Nutrients. 2020;12:1-13.

10. Murai IH, Fernandes AL, Sales LP, Pinto AJ, Goessler KF, Duran CS, et al. Effect of a single high dose of vitamin D3 on hospital length of stay in patients with moderate to severe COVID-19: a randomized clinical trial. JAMA. 2021;325:1053-1060.

11. Rhodes JM, Subramanian S, Laird E, Griffin G, Kenny RA. Perspective: vitamin D deficiency and COVID-19 severity - plausibly linked by latitude, ethnicity, impacts on cytokines, ACE2 and thrombosis. J Intern Med. 2021;289:97-115.

12. Nwosu BU, Maranda L, Berry R, Colocino B, Flores CD, Folkman K, et al. The vitamin D status of prison inmates. PLoS One. 2014;9:e90623.

13. Ambrosino I, Barbagelata E, Ortona E, Ruggieri A, Massiah G, Valerio-Giannico O, et al. Gender differences in patients with COVID-19: a narrative review. Monaldi Arch Chest Dis. 2020:90:318-324.

14. Macaya F, Espejo C, Valls A, Fernández-Ortiz A, González-del Castillo J, Martín-Sánchez FJ, et al. Interaction between age and vitamin d deficiency in severe covid-19 infection. Nutr Hosp. 2020;37:1039-1042.

15. Dramé $M$, Cofais $C$, Hentzien M, Proye $E$, Souleymane-Coulibaly $P$, Desprez A, et al. Relation between vitamin D and COVID-19 in aged people: a systematic review. Nutrients. 2021;13(4):1339.

16. Soto-Mota A, Marfil-GarzaBA, Martínez-RodríguezE, Barreto-RodríguezJO, López-Romo AE, Minutti PA, et al. The lownharm score for predicting mortality in patients diagnosed with COVID19: a multicentric validation study. J Am Coll Emerg Physicians Open. 2020;1:1436-1443.

17. Bacchetti P. Current sample size conventions: flaws, harms, and alternatives. BMC Med. 2010;8:1-7.

18. Bacchetti $P$, McCulloch CE, Segal MR. Simple, defensible sample sizes based on cost efficiency. Biometrics. 2008;64:577-585.

19. Holick MF, Binkley NC, Bischoff-Ferrari HA, Gordon CM, Hanley DA, Heaney RP, et al. Evaluation, treatment, and prevention of vitamin D deficiency: an endocrine society clinical practice guideline. J Clin Endocrinol Metab. 2011;96:1911-1930.

20. Álvarez-López JA, García-Contreras Al. Vitamina D y la pandemia por COVID-19. Rev Mex Endocrinol Metab Nutr. 2020;7.

21. Mariani J, Giménez VMM, Bergam I, et al. Association between vitamin $\mathrm{D}$ deficiency and COVID-19 incidence, complications, and mortality in 46 countries: An ecological study. Heal Secur. 2020;19:302-308.

22. Hars M, Mendes A, Serratrice C, Hermann FR, Gold G, Graf C, et al. Sex-specific association between vitamin D deficiency and COVID-19 mortality in older patients. Osteoporos Int. 2020;31:2495-2496. 
23. Lau FH, Majumder R, Torabi R, Saeg F, Hoffman R, Jeffrey D, et al. Vitamin $D$ insufficiency is prevalent in severe COVID-19. medRxiv. 2020

24. D'avolio A, Avataneo V, Manca A, Cusato J, de Nicolo A, Lucchini R, et al. 25-hydroxyvitamin $D$ concentrations are lower in patients with positive PCR for SARS-CoV-2. Nutrients. 2020:12:1359.

25. Baktash V, Hosack T, Patel N, et al. Vitamin D status and outcomes for hospitalised older patients with COVID-19. Postgrad Med J. 2021;97:442-447.

26. Meltzer DO, Best TJ, Zhang H, Vokes T, Arora V Solway J. Association of vitamin D status and other clinical characteristics with COVID-19 test results. JAMA Netw Open. 2020;3(9):e2019722. doi:10.1001/jamanetworkopen.2020.19722

27. Hastie CE, Mackay DF, Ho F, et al. Vitamin D concentrations and COVID-19 infection in UK Biobank. Diabetes Metab Syndr Clin Res Rev. 2020;14:561-565.

28. Peruzzu D, Pagano MT, Pierdominici M, Ruggieri A, Antinori A, D'Offizi G, et al. Synergy between vitamin $D$ and sex hormones in respiratory functionality of patients affected by COVID-19. Front Pharmacol. 2021:12:683529.
29. Pagano MT, Peruzzu D, Ruggieri A, Ortona E, Gagliardi MC. Vitamin D and sex differences in COVID-19. Front Endocrinol (Lausanne). 2020;11:567824.

30. Grant WB, Lahore H, McDonnell SL, Baggerly CA, French $\mathrm{CB}$, Aliano JL, et al. Evidence that vitamin D supplementation could reduce risk of Influenza and COVID-19 infections and deaths. Nutrients. 2020;12:988.

31. Whittemore PB. COVID-19 fatalities, latitude, sunlight, and vitamin D. Am J Infect Control. 2020;48:1042-1044.

32. Lanham-New SA, Webb AR, Cashman KD, Buttriss JL, Fallowfield JL, Masud T, et al. Vitamin D and SARS-CoV-2 virus/COVID-19 disease. BMJ Nutr Prev Health. 2020;3:106-110.

33. Skutsch M, Dobler C, McCall MBB, Fenner-Sanchez G. The association of UV with rates of COVID-19 transmission and deaths in Mexico: the possible mediating role of vitamin D. medRxiv. 2020.

34. Sattar N, Mclnnes IB, McMurray JJV. Obesity is a risk factor for severe COVID-19 infection: multiple potential mechanisms. Circulation. 2020;142(1):4-6. 